\title{
Fractional Fourier transform-based image encryption: phase retrieval algorithm
}

\author{
Bryan Hennelly, John T. Sheridan * \\ Department of Electronic and Electrical Engineering, Faculty of Engineering and Architecture, University College Dublin, \\ Belfield, Dublin 4, Ireland
}

Received 4 April 2003; received in revised form 25 July 2003; accepted 3 August 2003

\begin{abstract}
A number of methods have been proposed in the literature for the encryption of two-dimensional image information using optical systems based on the fractional Fourier transform (FRT). We present an image encryption technique, which is based on a recently proposed method of phase retrieval using the FRT. The optical implementation of the method is mentioned and digital simulations are presented. The technique is shown to be a powerful method of digital data and image encryption. In this paper, we outline the implementation of the algorithm and examine the sensitivities of the various encryption keys. We also compare the performance of the new technique to digital implementations of other FRT-based optical encryption schemes.
\end{abstract}

(c) 2003 Elsevier B.V. All rights reserved.

PACS: $42.30 ; 42.30 . \mathrm{K} ; 32.79 . \mathrm{S}$

Keywords: Fourier optics and optical signal processing: Fourier transforms; Fourier optics and optical signal processing: signal processing; Optical computing: information processing; Digital image processing; Phase retrieval

\section{Introduction}

The Fractional Fourier Transform (FRT) is a generalisation of the Fourier Transform (FT), which has received much attention in the literature [1-5]. The FT is a linear transformation, which allows a signal, originally captured in the position domain to be rotated through $90^{\circ}$ into the orthogonal spatial frequency domain. Thus, four successive applications of the FT $\left(360^{\circ}\right)$ are equivalent to the identity operation. In an analogous way, the FRT can be seen as a linear transformation $[2,3,6]$, which rotates the signal through any arbitrary angle into a mixed frequency - space domain. Optical implementations of the FRT using bulk optical elements have been developed [6,7]. The resulting system complexity is no more than that of the

\footnotetext{
${ }^{*}$ Corresponding author. Tel.: +353-1-716-1927; fax: +353-1-283-0921.

E-mail address: john.sheridan@ucd.ie (J.T. Sheridan).

$U R L:$ http://www.ucd.ie/eleceng.
} 
optical Fourier transform as it can be implemented with one or two lenses. The numerical implementation is not quite as straightforward and the optimum form of the discrete fractional Fourier transform (DFRT) has been the subject of debate in the literature $[8,9]$.

Information security has been receiving increasing attention in recent years. Because optical systems have the distinct advantage of processing two-dimensional (2D) complex data in parallel, thus carrying out otherwise relatively slow operations at great speeds, they are of growing interest for data encryption. A recently proposed FT-based optical encryption scheme [10] dubbed "double random phase encoding" involves multiplying by two random phases in the input and in the Fourier plane. If the random phases are statistically independent white noises then the encrypted image is also a white noise signal. The random phase key in the Fourier plane serves as the only key in this encryption scheme. The extra degrees of freedom available when using the FRT have been utilised as new keys in several recent encryption schemes [11-21].

One possible definition of the DFRT [8] has a correlation property which has been used to derive a recursive algorithm for the phase retrieval of a signal provided we have available the intensities of two fractional Fourier transforms of the original signal [22]. However, this 1D algorithm cannot be simply extended to include a second dimension, as significant non-trivial differences occur in going from 1D to 2D. In this paper, we extend this algorithm to two dimensions and we use it to encrypt a standard image. We also point out difficulties that arise starting with the original 1D phase retrieval algorithm [22]. We show that through the use of two random phase keys and the intensities of our image we can obtain a highly encrypted image. Decryption is in the form of 2D phase retrieval. Many of FRT encryption algorithms also employ the use of one or more random phase keys [11-21]. Random phase keys can also be used with other optically implemented transforms to encrypt data [24].

The outline of this paper is as follows: in Section 2, we briefly recall the continuous FRT and in Section 3, we describe its discrete counterpart and present some of its properties. In Section 4, we discuss the phase retrieval algorithm and its application to encryption. In Section 5, we present results for our algorithm and show the sensitivities of the various keys. We compare the sensitivities of these keys to those of recent encryption algorithms. In Section 6, we present our conclusions and in Appendices A and B, we describe the correlation property proof and why the phase retrieval algorithm does not break down when extended to two dimensions.

\section{Fractional Fourier transform}

Conventionally, the ath order FRT, $f_{a}\left(x_{a}\right)$, of a function $f_{0}\left(x_{0}\right)$ is calculated using integral transform kernel given by $[3,4]$

$$
K_{a}\left(x, x_{a}\right)= \begin{cases}A_{\phi} \exp \left\{\mathrm{j} \pi\left(x^{2} \cot \phi-2 x x_{a}+x_{a}^{2} \cot \phi\right)\right\}, & 0<|a|<2, \\ \delta\left(x-x_{a}\right), & a=0, \\ \delta\left(x+x_{a}\right), & a= \pm 2\end{cases}
$$

where

$$
A \phi=\exp [-\mathrm{j} \pi \operatorname{sgn}(\sin \phi) / 4+\mathrm{j} \phi / 2] \text { and } \phi=a \pi / 2
$$

and $x$ and $x_{a}$ represent the coordinate systems for the input or zeroth order domain and output $a$ th fractional domain, respectively. The FRT is linear and has the property that it is index additive,

$$
F_{a}\left\{F_{b}\{f(x)\}\right\}=F_{a+b}\{f(x)\} .
$$

It is possible to extend the definition of the FRT order beyond \pm 2

$$
F_{a}\{f(x)\}=F_{a+4 n}\{f(x)\} \quad \forall n=\text { integer. }
$$

In image encryption, we will of course be dealing with two-dimensional signals. The 2D FRT has separable kernels in both dimensions and so the above definition can be naturally extended [6]. 


\section{The discrete fractional Fourier transform}

Numerical implementation of the fractional Fourier transform has been the subject of much discussion $[8,9]$. Arguably the most plausible definition of the DFRT to date is outlined in [9] but this definition has not been implemented as a fast algorithm. Another definition which does not appear to satisfy all of the requirements for the discrete definition [9], but which is an effective method of computing the continuous FRT, will be used in this paper. It is defined by sampling the kernel of the FRT at appropriate sampling intervals [23]. In [8] a fast algorithm to calculate this definition of the DFRT is presented. However, this method requires a slightly different sampling criterion, which causes it to be non-unitary, and thus cannot be used in the following analysis.

Here, the 2D DFRT is defined as follows:

$$
\begin{aligned}
f_{p_{x}, p_{y}}\left(m_{x} \Delta x_{p_{x}}, m_{y} \Delta y_{p_{y}},\right) \\
=F^{p_{x}, p_{y}}\left\{f_{0,0}\left(l_{x} \Delta x_{0}, l_{y} \Delta y_{0}\right)\right\}\left(m_{x} \Delta x_{p_{x}}, m_{y} \Delta y_{p_{y}}\right) \\
=A_{p_{x}} A_{p_{y}} \Delta x_{0} \Delta y_{0} \sum_{l_{y}=\frac{-N_{y}}{2}}^{\frac{N_{y}}{2}-1} \sum_{l_{x}=\frac{-N_{x}}{2}}^{\frac{N_{x}}{2}-1}\left[f_{0,0}\left(l_{x} \Delta x_{0}, l_{y} \Delta y_{0}\right) \exp \left\{\mathrm{j} \pi \cot \left(\frac{p_{x} \pi}{2}\right)\left[\left(l_{x} \Delta x_{0}\right)^{2}+\left(m_{x} \Delta x_{p_{x}}\right)^{2}\right]-\mathrm{j} 2 \pi \frac{l_{x} m_{x}}{N_{x}}\right\}\right. \\
\left.\quad \times \exp \left\{\mathrm{j} \pi \cot \left(\frac{p_{y} \pi}{2}\right)\left[\left(l_{x} \Delta y_{0}\right)^{2}+\left(m_{y} \Delta y_{p_{y}}\right)^{2}\right]-\mathrm{j} 2 \pi \frac{l_{y} m_{y}}{N_{y}}\right\}\right]
\end{aligned}
$$

where $f_{0,0}\left(l_{x} \Delta x_{0}, l_{y} \Delta y_{0}\right)$ is the discrete function which we transform, and the integers $l_{x}$ and $l_{y}$ have the following ranges

$$
-\frac{N_{x}}{2} \leqslant l_{x} \leqslant \frac{N_{x}}{2}-1 \text { and } \quad-\frac{N_{y}}{2} \leqslant l_{y} \leqslant \frac{N_{y}}{2}-1 .
$$

$\Delta x_{0}$ and $\Delta y_{0}$ are the sampling intervals of our input function in the $x$ - and $y$-directions, respectively, $\Delta x_{p_{x}}$ and $\Delta y_{p_{y}}$ are the sampling intervals in the new FRT domain. They are defined below in Eq. (8). Both $m_{x}$ and $m_{y}$ have the same range of values as $l_{x}$ and $l_{y}$.

The original representation can be retrieved from the $p$ th order FRT using the inverse relationship

$$
\begin{aligned}
f_{0,0}( & \left.n_{x} \Delta x_{0}, n_{y} \Delta y_{0}\right) \\
= & F^{-p_{x},-p_{y}}\left\{f_{p_{x}, p_{y}}\left(m_{x} \Delta x_{p_{x}}, m_{y} \Delta y_{p_{y}}\right)\right\}\left(n_{x} \Delta x_{0}, n_{y} \Delta y_{0}\right) \\
= & A_{-p_{x}} A_{-p_{y}} \Delta x_{p_{x}} \Delta y_{p_{y}} \sum_{m_{y}=\frac{N_{y}}{2}}^{\frac{N_{y}}{2}-1} \sum_{m_{x}=\frac{-N_{x}}{2}}^{\frac{N_{x}}{2}-1}\left[f_{p_{x}, p_{y}}\left(m_{x} \Delta x_{p_{x}}, m_{y} \Delta y_{p_{y}}\right) \exp \left\{-\mathrm{j} \pi \cot \left(\frac{p_{x} \pi}{2}\right)\left[\left(n_{x} \Delta x_{0}\right)^{2}+\left(m_{x} \Delta x_{p_{x}}\right)^{2}\right]\right\}\right. \\
& \left.\times \exp \left\{\mathrm{j} 2 \pi \frac{n_{x} m_{x}}{N_{x}}\right\} \exp \left\{-\mathrm{j} \pi \cot \left(\frac{p_{y} \pi}{2}\right)\left[\left(n_{y} \Delta y_{0}\right)^{2}+\left(m_{y} \Delta y_{p_{y}}\right)^{2}\right]\right\} \exp \left\{\mathrm{j} 2 \pi \frac{n_{y} m_{y}}{N_{y}}\right\}\right]
\end{aligned}
$$

From digital signal theory [23] we can determine the following sampling criteria,

$$
\Delta x_{p_{x}}=\frac{\sin \left(\frac{p_{x} \pi}{2}\right)}{N_{x} \Delta x_{0}}, \quad \Delta y_{p_{y}}=\frac{\sin \left(\frac{p_{y} \pi}{2}\right)}{N_{y} \Delta y_{0}} .
$$

Then, using the above as our discrete model of the FRT, the following correlation property can be defined 


$$
\begin{aligned}
\sum_{l_{y}=-\frac{N}{2}}^{\frac{N_{y}}{2}-1} \sum_{l_{x}=-\frac{N}{2}}^{\frac{N_{y}}{2}-1}[ & f_{0,0}^{*}\left(l_{x} \Delta x_{0}, l_{y} \Delta y_{0}\right) f_{0,0}\left[\left(l_{x}+k_{x}\right) \Delta x_{0},\left(l_{y}+k_{y}\right) \Delta y_{0}\right] \exp \left\{\mathrm{j} 2 \pi \cot \left(\frac{p_{x} \pi}{2}\right) l_{x} k_{x}\left(\Delta x_{0}\right)^{2}\right\} \\
& \times \exp \left\{\mathrm{j} 2 \pi \cot \left(\frac{p_{y} \pi}{2}\right) l_{y} k_{y}\left(\Delta y_{0}\right)^{2}\right\}=\frac{\left|\sin \left(\frac{p_{x} \pi}{2}\right)\right|}{N_{x} \Delta x_{0}^{2}} \frac{\left|\sin \left(\frac{p_{y} \pi}{2}\right)\right|}{N_{y} \Delta y_{0}^{2}} \\
& \times \exp \left\{\mathrm{j} \pi \cot \left(\frac{p_{x} \pi}{2}\right) k_{x}^{2}\left(\Delta x_{0}\right)^{2}\right\} \exp \left\{\mathrm{j} \pi \cot \left(\frac{p_{y} \pi}{2}\right) k_{y}^{2}\left(\Delta y_{0}\right)^{2}\right\} \\
& \left.\times \sum_{m_{y}=\frac{N_{y}}{2}}^{\frac{N_{y}}{2}-1} \sum_{m_{x}=\frac{N_{x}}{2}}^{\frac{N_{x}}{2}-1}\left|f_{p_{x}, p_{y}}\left(m_{x} \Delta x_{p_{x}}, m_{y} \Delta y_{p_{y}}\right)\right|^{2} \exp \left\{\mathrm{j} 2 \pi \frac{k_{y} m_{y}}{N_{y}}\right\} \exp \left\{\mathrm{j} 2 \pi \frac{k_{y} m_{y}}{N_{y}}\right\}\right] .
\end{aligned}
$$

We offer a proof of this property in Appendix A.

We now make use of this correlation property in an analogous way to that done in [22].

\section{Encryption/decryption algorithms}

In this section, we define our encryption and decryption procedures. Encryption is carried out using two random phase keys and two FRT operations of arbitrary order. We apply one of the phase planes followed by one of the FRT operations to our input image signal and store only the intensity of the result. We repeat this procedure on our input image signal using the second phase key and applying a second FRT operation. We note that both of these FRT operations may have different orders in the two orthogonal dimensions. In order to decrypt our image, we must know both phase keys and the orders of the two FRT operations, comprising of a total of four FRT order keys (if we take the orders to be different in the $x$ and $y$-directions). Decryption is based on a correlation property of the FRT that allows us to recursively recover our image.

\subsection{Encryption}

We begin with our signal to be encrypted $g_{0,0}\left(l_{x} \Delta x_{0}, l_{y} \Delta y_{0}\right)$. We note that this signal can be complex, containing both intensity and phase information. Therefore, it can contain the information of two images where the intensity of the signal can represent one image and the phase of our signal can be modulated to represent a second image.

We intend to encrypt this data using two FRTs and two random phase keys in the form of two statistically independent white sequences $\exp \left\{\mathrm{j} \phi\left(l_{x} \Delta x_{0}, l_{y} \Delta y_{0}\right)\right\}$ and $\exp \left\{\mathrm{j} \varphi\left(l_{x} \Delta x_{0}, l_{y} \Delta y_{0}\right)\right\}$, where $\phi\left(l_{x} \Delta x_{0}\right.$, $\left.l_{y} \Delta y_{0}\right)$ and $\varphi\left(l_{x} \Delta x_{0}, l_{y} \Delta y_{0}\right)$ are uniformly distributed in $[0,2 \pi]$. The keys necessary to decrypt the signal will be the two random phase keys and the two sets of fractional orders, in both $x$ and $y$ used during the encryption process adding four more encryption keys and giving us a total of six keys.

To encrypt our image, we carry out the following. We multiply our input signal by the first random phase to give us

$$
f 1_{0,0}\left(l_{x} \Delta x_{0}, l_{y} \Delta y_{0}\right)=g_{0,0}\left(l_{x} \Delta x_{0}, l_{y} \Delta y_{0}\right) \exp \left\{\mathrm{j} \phi\left(l_{x} \Delta x_{0}, l_{y} \Delta y_{0}\right)\right\} .
$$

Applying a DFRT operation of order $p_{x}$ in the $x$-direction and $p_{y}$ in the $y$-direction to this result gives,

$$
f 1_{p_{x}, p_{y}}\left(m_{x} \Delta x_{p_{x}}, m_{y} \Delta y_{p_{y}}\right)=F^{p_{x}, p_{y}}\left\{g_{0,0}\left(l_{x} \Delta x_{0}, l_{y} \Delta y_{0}\right) \exp \left\{\mathrm{j} \phi\left(l_{x} \Delta x_{0}, l_{y} \Delta y_{0}\right)\right\}\right\} .
$$


We store only the intensity of this signal $\left|f 1_{p_{x}, p_{y}}\left(m_{x} \Delta x_{p_{x}}, m_{y} \Delta y_{p_{y}}\right)\right|^{2}$ to give us half of our required encrypted data. We note that $l_{x}$ and $l_{y}$ have values in the range given by Eq. (6). However, the decryption procedure which will be described shortly requires that this intensity has twice as many samples in each dimension

$$
\Delta x_{p_{x}}=\frac{\sin \left(\frac{p_{x} \pi}{2}\right)}{2 N_{x} \Delta x_{0}}, \quad \Delta y_{p_{y}}=\frac{\sin \left(\frac{p_{y} \pi}{2}\right)}{2 N_{y} \Delta y_{0}},
$$

where $m_{x}$ and $m_{y}$ have the following ranges of values

$$
-N_{x} \leqslant m_{x} \leqslant N_{x}-1 \text { and }-N_{y} \leqslant m_{y} \leqslant N_{y}-1 .
$$

In other words, we require our transformed function to be sampled at double the rate necessary to describe our input function completely. We can obtain this interpolated function from our input function $f 1_{0,0}\left(l_{x} \Delta x_{0}, l_{y} \Delta y_{0}\right)$ by zero padding $f 1_{0,0}\left(l_{x} \Delta x_{0}, l_{y} \Delta y_{0}\right)$ so that we extend the range of $l_{x}$ and $l_{y}$ to

$$
-N_{x} \leqslant l_{x} \leqslant N_{x}-1 \text { and }-N_{y} \leqslant l_{y} \leqslant N_{y}-1
$$

including zeros for the function values in the ranges

$$
-N_{x} \leqslant l_{x} \leqslant-\frac{N_{x}}{2}-1, \quad \frac{N_{x}}{2} \leqslant l_{x} \leqslant N_{x}-1
$$

and

$$
-N_{y} \leqslant l_{y} \leqslant-\frac{N_{y}}{2}-1, \quad \frac{N_{y}}{2} \leqslant l_{y} \leqslant N_{y}-1 .
$$

We then carry out our FRT operations on this padded input function:

$$
\begin{aligned}
f 1_{p_{x}, p_{y}}\left(m_{x} 2 \Delta x_{p_{x}}, m_{y} 2 \Delta y_{p_{y}}\right)= & A_{p_{x}} A_{p_{y}} \Delta x_{0} \Delta y_{0} \sum_{l_{y}=\frac{-N_{y}}{2}}^{\frac{N_{y}}{2}-1} \sum_{l_{x}=\frac{-N_{x}}{2}}^{\frac{N_{x}}{2}-1}\left[f 1_{0,0}\left(l_{x} \Delta x_{0}, l_{y} \Delta y_{0}\right)\right. \\
& \times \exp \left\{\mathrm{j} \pi \cot \left(\frac{p_{x} \pi}{2}\right)\left[\left(l_{x} \Delta x_{0}\right)^{2}+\left(m_{x} 2 \Delta x_{p_{x}}\right)^{2}\right]\right\} \exp \left\{-\mathrm{j} 2 \pi \frac{l_{x} m_{x}}{N_{x}}\right\} \\
& \left.\times \exp \left\{\mathrm{j} \pi \cot \left(\frac{p_{y} \pi}{2}\right)\left[\left(l_{y} \Delta y_{0}\right)^{2}+\left(m_{y} 2 \Delta y_{p_{y}}\right)^{2}\right]\right\} \exp \left\{-\mathrm{j} 2 \pi \frac{l_{y} m_{y}}{N_{y}}\right\}\right] .
\end{aligned}
$$

If we take $f 1_{0,0}\left(l_{x} \Delta x_{0}, l_{y} \Delta y_{0}\right)$ to have zero values in the ranges given by (15) we can write

$$
\begin{aligned}
f 1_{p_{x}, p_{y}}\left(m_{x} \Delta x_{p_{x}}, m_{y} \Delta y_{p_{y}}\right)= & A_{p_{x}} A_{p_{y}} \Delta x_{0} \Delta y_{0} \sum_{l_{y}=N_{y}}^{N_{y}-1} \sum_{l_{x}=N_{x}}^{N_{x}-1}\left[f 1_{0,0}\left(l_{x} \Delta x_{0}, l_{y} \Delta y_{0}\right)\right. \\
& \times \exp \left\{\mathrm{j} \pi \cot \left(\frac{p_{x} \pi}{2}\right)\left[\left(l_{x} \Delta x_{0}\right)^{2}+\left(m_{x} \Delta x_{p_{x}}\right)^{2}\right]\right\} \exp \left\{-\mathrm{j} 2 \pi \frac{l_{x} m_{x}}{N_{x}}\right\} \\
& \left.\times \exp \left\{\mathrm{j} \pi \cot \left(\frac{p_{y} \pi}{2}\right)\left[\left(l_{y} \Delta y_{0}\right)^{2}+\left(m_{y} \Delta y_{p_{y}}\right)^{2}\right]\right\} \exp \left\{-\mathrm{j} 2 \pi \frac{l_{y} m_{y}}{N_{y}}\right\}\right] .
\end{aligned}
$$

To obtain the second half of our encrypted data, we carry out a similar procedure, this time applying the second random phase in a different fractional domain. We multiply our input signal by the second random phase to give us

$$
f 2_{0,0}\left(l_{x} \Delta x_{0}, l_{y} \Delta y_{0}\right)=g_{0,0}\left(l_{x} \Delta x_{0}, l_{y} \Delta y_{0}\right) \exp \left\{\mathrm{j} \varphi\left(l_{x} \Delta x_{0}, l_{y} \Delta y_{0}\right)\right\}
$$

and carry out a DFRT operation of order $q_{x}$ in the $x$-direction and $q_{y}$ in the $y$-direction. This gives, 


$$
f 2_{q_{x}, q_{y}}\left(m_{x} \Delta x_{q_{x}}, m_{y} \Delta y_{q_{y}}\right)=F^{q_{x}, q_{y}}\left\{g_{0,0}\left(l_{x} \Delta x_{0}, l_{y} \Delta y_{0}\right) \exp \left\{\mathrm{j} \varphi\left(l_{x} \Delta x_{0}, l_{y} \Delta y_{0}\right)\right\}\right\} .
$$

Once again we capture only the intensity of this signal $\left|f 2_{q_{x}, q_{y}}\left(m_{x} \Delta x_{q_{x}}, m_{y} \Delta y_{q_{y}}\right)\right|^{2} \cdot f 2_{q_{x}, q_{y}}\left(m_{x} \Delta x_{q_{x}}, m_{y} \Delta y_{q_{y}}\right)$ is then defined in an identical manner to $f 1_{p_{x}, p_{y}}\left(m_{x} \Delta x_{p_{x}}, m_{y} \Delta y_{p_{y}}\right)$ using:

$$
\begin{aligned}
f 2_{q_{x}, q_{y}}\left(m_{x} \Delta x_{q_{x}}, m_{y} \Delta y_{q_{y}}\right)= & A_{q_{x}} A_{q_{y}} \Delta x_{0} \Delta y_{0} \sum_{l_{y}=N_{y}}^{N_{y}-1} \sum_{l_{x}=N_{x}}^{N_{x}-1}\left[f 1_{0,0}\left(l_{x} \Delta x_{0}, l_{y} \Delta y_{0}\right)\right. \\
& \times \exp \left\{\mathrm{j} \pi \cot \left(\frac{q_{x} \pi}{2}\right)\left[\left(l_{x} \Delta x_{0}\right)^{2}+\left(m_{x} \Delta x_{q_{x}}\right)^{2}\right]\right\} \\
& \times \exp \left\{-\mathrm{j} 2 \pi \frac{l_{x} m_{x}}{N_{x}}\right\} \exp \left\{\mathrm{j} \pi \cot \left(\frac{q_{y} \pi}{2}\right)\left[\left(l_{y} \Delta y_{0}\right)^{2}+\left(m_{y} \Delta y_{q_{y}}\right)^{2}\right]\right\} \\
& \left.\times \exp \left\{-\mathrm{j} 2 \pi \frac{l_{y} m_{y}}{N_{y}}\right\}\right] .
\end{aligned}
$$

We represent this encryption scheme using a block diagram flowchart in Fig. 1.

We note that if the procedure were to be carried out optically, the random phase planes might be implemented using Spatial Light Modulators [21], the FRT operations would be implemented using free space and lenses [7], and we could use a CCD camera to capture the intensity of the resultant wave fields.

\subsection{Decryption}

We wish to recover our original signal (phase and intensity) from the two intensities generated above. We will make use of the correlation property given in Eq. (9). We apply this property to both of the intensities. In this way, we can derive a recursive algorithm to determine each value of our original signal. Thus we can recover the original signals phase and amplitude.

Noting that $f 1_{0,0}\left(l_{x} \Delta x_{0}, l_{y} \Delta y_{0}\right)$ and $f 2_{0,0}\left(l_{x} \Delta x_{0}, l_{y} \Delta y_{0}\right)$ have both been padded with zero values outside the range of interest and using the correlation property listed above we can now define the following two functions:

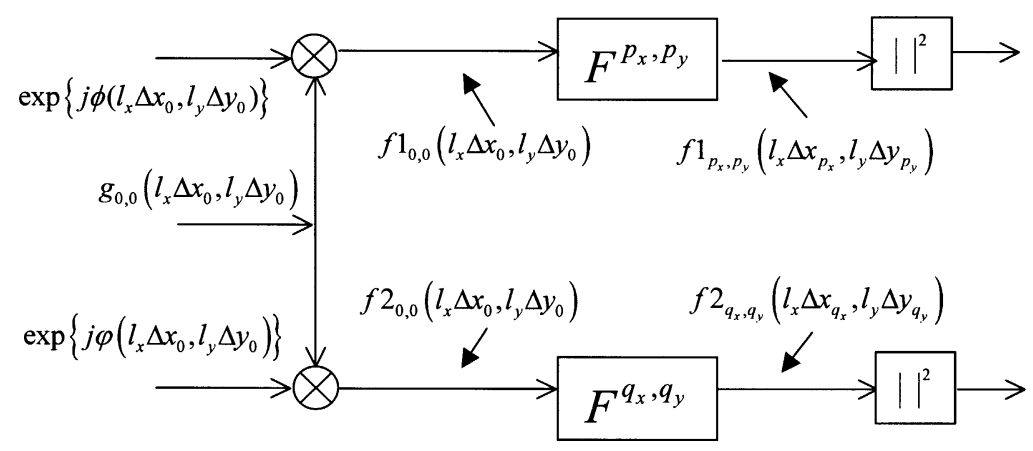

Fig. 1. The encryption procedure. 


$$
\begin{aligned}
\operatorname{RHS1}\left(k_{x}, k_{y}\right)= & \sum_{l_{y}=-N_{y}}^{\frac{N_{y}}{2}-k_{x}-1} \sum_{l_{x}=-N_{x}}^{\frac{N_{y}}{2}-k_{y}-1}\left[f 1_{0,0}^{*}\left(l_{x} \Delta x_{0}, l_{y} \Delta y_{0}\right) f 1_{0,0}\left[\left(l_{x}+k_{x}\right) \Delta x_{0},\left(l_{y}+k_{y}\right) \Delta y_{0}\right]\right. \\
& \left.\times \exp \left\{\mathrm{j} 2 \pi \cot \left(\frac{p_{x} \pi}{2}\right) l_{x} k_{x}\left(\Delta x_{0}\right)^{2}\right\} \exp \left\{\mathrm{j} 2 \pi \cot \left(\frac{p_{y} \pi}{2}\right) l_{y} k_{y}\left(\Delta y_{0}\right)^{2}\right\}\right] \\
= & \frac{\left|\sin \left(\frac{p_{x} \pi}{2}\right)\right|}{2 N_{x} \Delta x_{0}^{2}} \frac{\left|\sin \left(\frac{p_{y} \pi}{2}\right)\right|}{2 N_{y} \Delta y_{0}^{2}} \exp \left\{\mathrm{j} \pi \cot \left(\frac{p_{x} \pi}{2}\right) k_{x}^{2}\left(\Delta x_{0}\right)^{2}\right\} \exp \left\{\mathrm{j} \pi \cot \left(\frac{p_{y} \pi}{2}\right) k_{y}^{2}\left(\Delta y_{0}\right)^{2}\right\} \\
\operatorname{RHS} 2\left(k_{x}, k_{y}\right)= & \sum_{l_{y}=-N_{y}}^{\frac{N_{y}}{2}-k_{x}-1} \sum_{l_{x}=-N_{x}}^{\frac{N_{y}}{2}-k_{y}-1}\left[f 2_{0,0}^{*}\left(l_{x} \Delta x_{0}, l_{y} \Delta y_{0}\right) f 2_{0,0}\left[\left(l_{x}+k_{x}\right) \Delta x_{0},\left(l_{y}+k_{y}\right) \Delta y_{0}\right]\right. \\
& \times \sum_{m_{y}=-N_{x}}^{N_{y}-1}\left|f 1_{p_{x}, p_{y}}\left(m_{x} \Delta x_{p_{x}}, m_{y} \Delta y_{p_{y}}\right)\right|^{2} \exp \left\{\mathrm{j} 2 \pi \frac{k_{x} m_{x}}{N_{x}}\right\} \exp \left\{\mathrm{j} 2 \pi \frac{k_{x} m_{y}}{N_{y}}\right\} \\
= & \frac{\left|\sin \left(\frac{q_{x} \pi}{2}\right)\right|}{2 N_{x} \Delta x_{0}^{2}} \frac{\left|\sin \left(\frac{q_{y} \pi}{2}\right)\right|}{2 N_{y} \Delta y_{0}^{2}} \exp \left\{\mathrm{j} \pi \cot \left(\frac{q_{x} \pi}{2}\right) l_{x} k_{x}\left(\Delta x_{0}\right)^{2}\right\} \exp \left\{\mathrm{j} 2 \pi \cot \left(\frac{q_{y} \pi}{2}\right) n_{y} k_{y}\left(\Delta y_{0}\right)^{2}\right\} \\
& \left.\quad \times \sum_{m_{y}=-N_{y}}^{N_{y}-1} \sum_{m_{x}=-N_{x}}^{N_{x}-1}\left|f 2_{q_{x}, q_{y}}\left(m_{x} \Delta x_{q_{x}}, m_{y} \Delta y_{q_{y}}\right)\right|^{2} \exp \left\{\mathrm{j} 2 \pi \frac{k_{x} m_{x}}{N_{x}}\right\} \operatorname{j} \pi \cot \left(\frac{q_{y} \pi}{2}\right) k_{y}^{2}\left(\Delta y_{0}\right)^{2}\right\}
\end{aligned}
$$

We note that

$$
f 2_{0,0}\left(l_{x} \Delta x_{0}, l_{y} \Delta y_{0}\right)=f 1_{0,0}\left(l_{x} \Delta x_{0}, l_{y} \Delta y_{0}\right) \exp \left\{\mathrm{j} \gamma\left(l_{x} \Delta x_{0}, l_{y} \Delta y_{0}\right)\right\}
$$

where

$$
\gamma\left(l_{x} \Delta x_{0}, l_{y} \Delta y_{0}\right)=\varphi\left(l_{x} \Delta x_{0}, l_{y} \Delta y_{0}\right)-\phi\left(l_{x} \Delta x_{0}, l_{y} \Delta y_{0}\right) .
$$

Introducing the integer variable $h_{x}$ and $h_{y}$ where

$$
\begin{aligned}
& h_{x}=N_{x}-k_{x} \Rightarrow k_{x}=N_{x}-h_{x}, \\
& h_{y}=N_{y}-k_{y} \Rightarrow k_{y}=N_{y}-h_{y}
\end{aligned}
$$

we can now write the above Eqs. (20) and (21) with these substitutions

$$
\begin{aligned}
\operatorname{RHS} 1\left(N_{x}-h_{x}, N_{y}-h_{y}\right)= & \sum_{l_{y}=-N_{y}}^{\left(-\frac{N_{y}}{2}+h_{y}-1\right)} \sum_{l_{x}=-N_{x}}^{\left(-\frac{N_{x}}{2}+h_{x}-1\right)}\left[f 1_{0,0}^{*}\left(l_{x} \Delta x_{0}, l_{y} \Delta y_{0}\right) f 1_{0,0}\right. \\
& \times\left[\left(l_{x}+N_{x}-h_{x}\right) \Delta x_{0},\left(l_{y}+N_{y}-h_{y}\right) \Delta y_{0}\right] \exp \left\{\mathrm{j} 2 \pi \cot \left(\frac{p_{x} \pi}{2}\right) l_{x}\left(N_{x}-h_{x}\right)\left(\Delta x_{0}\right)^{2}\right\} \\
& \left.\times \exp \left\{\mathrm{j} 2 \pi \cot \left(\frac{p_{y} \pi}{2}\right) l_{y}\left(N_{y}-h_{y}\right)\left(\Delta y_{0}\right)^{2}\right\}\right]
\end{aligned}
$$


and

$$
\begin{aligned}
\operatorname{RHS} 2\left(N_{x}-h_{x}, N_{y}-h_{y}\right)= & \sum_{l_{y}=-N_{y}}^{\left(-\frac{N_{y}}{2}+h_{y}-1\right)} \sum_{l_{x}=-N_{x}}^{\left(-\frac{N_{x}}{2}+h_{x}-1\right)}\left[f 1_{0,0}^{*}\left(l_{x} \Delta x_{0}, l_{y} \Delta y_{0}\right)\right. \\
& \times \exp \left\{-\mathrm{j} \gamma\left(l_{x} \Delta x_{0}, l_{y} \Delta y_{0}\right)\right\} f 1_{0,0}\left[\left(l_{x}+N_{x}-h_{x}\right) \Delta x_{0},\left(l_{y}+N_{y}-h_{y}\right) \Delta y_{0}\right] \\
& \times \exp \left\{\mathrm{j} \gamma\left[\left(l_{x}+N_{x}-h_{x}\right) \Delta x_{0},\left(l_{y}+N_{y}-h_{y}\right) \Delta y_{0}\right]\right\} \\
& \times \exp \left\{\mathrm{j} 2 \pi \cot \left(\frac{q_{x} \pi}{2}\right) l_{x}\left(N_{x}-h_{x}\right)\left(\Delta x_{0}\right)^{2}\right\} \exp \left\{\mathrm{j} 2 \pi \cot \left(\frac{q_{y} \pi}{2}\right) l_{y}\right. \\
& \left.\left.\times\left(N_{y}-h_{y}\right)\left(\Delta y_{0}\right)^{2}\right\}\right] .
\end{aligned}
$$

We will use Eqs. (25) and (26) to find two sets of products of the type

$$
\begin{aligned}
& f 1_{0,0}^{*}\left(\left(\frac{-N_{x}}{2}\right) \Delta x_{0},\left(\frac{-N_{x}}{2}\right) \Delta y_{0}\right) f 1_{0,0}\left[\left(\frac{N_{x}}{2}-h_{x}\right) \Delta x_{0},\left(\frac{N_{y}}{2}-h_{y}\right) \Delta y_{0}\right], \\
& f 1_{0,0}^{*}\left(\left(\frac{-N_{x}}{2}+h_{x}-1\right) \Delta x_{0},\left(\frac{-N_{x}}{2}+h_{y}-1\right) \Delta y_{0}\right) f 1_{0,0}\left[\left(\frac{N_{x}}{2}-1\right) \Delta x_{0},\left(\frac{N_{y}}{2}-1\right) \Delta y_{0}\right],
\end{aligned}
$$

where $h_{x}$ takes the values range $1 \leqslant h_{x} \leqslant \frac{N_{x}}{2}+1$ and similarly we $h_{y}$ takes the value range from $1 \leqslant h_{y} \leqslant \frac{N_{y}}{2}+1$. We will examine the cases $\left(h_{x}=1, h_{y}=1\right),\left(h_{x}=1, h_{y}=2\right)$, and $\left(h_{x}=2, h_{y}=1\right)$ separately and then define a more general recursive formula for all other values of $h_{x}$ and $h_{y}$.

Examining Eq. (25) and taking $\left(h_{x}=1, h_{y}=1\right)$, we see that

$$
\begin{aligned}
f 1_{0,0}^{*} & \left(\left(\frac{-N_{x}}{2}\right) \Delta x_{0},\left(\frac{-N_{y}}{2}\right) \Delta y_{0}\right) f 1_{0,0}\left[\left(\frac{N_{x}}{2}-1\right) \Delta x_{0},\left(\frac{N_{y}}{2}-1\right) \Delta y_{0}\right] \\
= & \operatorname{RHS1}\left(N_{x}-1, N_{y}-1\right) \exp \left\{\mathrm{j} 2 \pi \cot \left(\frac{p_{x} \pi}{2}\right)\left(\frac{N_{x}}{2}\right)\left(N_{x}-1\right)\left(\Delta x_{0}\right)^{2}\right\} \\
& \times \exp \left\{\mathrm{j} 2 \pi \cot \left(\frac{p_{y} \pi}{2}\right)\left(\frac{N_{y}}{2}\right)\left(N_{y}-1\right)\left(\Delta y_{0}\right)^{2}\right\} .
\end{aligned}
$$

Eq. (26) provides us with a similar result

$$
\begin{aligned}
& f 1_{0,0}^{*}\left(\left(\frac{-N_{x}}{2}\right) \Delta x_{0},\left(\frac{-N_{y}}{2}\right) \Delta y_{0}\right) f 1_{0,0}\left[\left(\frac{N_{x}}{2}-1\right) \Delta x_{0},\left(\frac{N_{y}}{2}-1\right) \Delta y_{0}\right] \\
& =\operatorname{RHS} 2\left(N_{x}-1, N_{y}-1\right) \exp \left\{\mathrm{j} 2 \pi \cot \left(\frac{p_{x} \pi}{2}\right)\left(\frac{N_{x}}{2}\right)\left(N_{x}-1\right)\left(\Delta x_{0}\right)^{2}\right\} \\
& \quad \times \exp \left\{\mathrm{j} 2 \pi \cot \left(\frac{p_{y} \pi}{2}\right)\left(\frac{N_{y}}{2}\right)\left(N_{y}-1\right)\left(\Delta y_{0}\right)^{2}\right\} \exp \left\{\mathrm{j} \gamma\left(\left(\frac{-N_{x}}{2}\right) \Delta x_{0},\left(\frac{-N_{y}}{2}\right) \Delta y_{0}\right)\right\} \\
& \quad \times \exp \left\{-\mathrm{j} \gamma\left(\left(\frac{N_{x}}{2}-1\right) \Delta x_{0},\left(\frac{N_{y}}{2}-1\right) \Delta y_{0}\right)\right\}, \\
& a\left(h_{x}, h_{y}\right)=\exp \left\{-\mathrm{j} \pi \cot \left(\frac{p_{x} \pi}{2}\right) N_{x}\left(N_{x}-h_{x}\right)\left(\Delta x_{0}\right)^{2}\right\} \exp \left\{-\mathrm{j} \pi \cot \left(\frac{p_{y} \pi}{2}\right) N_{y}\left(N_{y}-h_{y}\right)\left(\Delta y_{0}\right)^{2}\right\}, \\
& b\left(h_{x}, h_{y}\right)=\exp \left\{-\mathrm{j} \pi \cot \left(\frac{p_{x} \pi}{2}\right)\left(N_{x}-2 h_{x}+2\right)\left(N_{x}-h_{x}\right)\left(\Delta x_{0}\right)^{2}\right\} \\
& \quad \times \exp \left\{-\mathrm{j} \pi \cot \left(\frac{p_{y} \pi}{2}\right)\left(N_{y}-2 h_{y}+2\right)\left(N_{y}-h_{y}\right)\left(\Delta y_{0}\right)^{2}\right\},
\end{aligned}
$$




$$
\begin{aligned}
c\left(h_{x}, h_{y}\right)= & \exp \left\{-\mathrm{j} \pi \cot \left(\frac{q_{x} \pi}{2}\right) N_{x}\left(N_{x}-h_{x}\right)\left(\Delta x_{0}\right)^{2}\right\} \exp \left\{-\mathrm{j} \pi \cot \left(\frac{q_{y} \pi}{2}\right) N_{y}\left(N_{y}-h_{y}\right)\left(\Delta y_{0}\right)^{2}\right\} \\
& \times \exp \left\{-\mathrm{j} \gamma\left(\left(\frac{-N_{x}}{2}\right) \Delta x_{0},\left(\frac{-N_{y}}{2}\right) \Delta y_{0}\right)\right\} \exp \left\{\mathrm{j} \gamma\left(\left(\frac{N_{x}}{2}-h_{x}\right) \Delta x_{0},\left(\frac{N_{y}}{2}-h_{y}\right) \Delta y_{0}\right)\right\} \\
d\left(h_{x}, h_{y}\right)= & \exp \left\{-\mathrm{j} \pi \cot \left(\frac{q_{x} \pi}{2}\right)\left(N_{x}-2 h_{x}+2\right)\left(N_{x}-h_{x}\right)\left(\Delta x_{0}\right)^{2}\right\} \exp \left\{-\mathrm{j} \pi \cot \left(\frac{q_{y} \pi}{2}\right)\right. \\
& \left.\times\left(N_{y}-2 h_{y}+2\right)\left(N_{y}-h_{y}\right)\left(\Delta y_{0}\right)^{2}\right\} \exp \left\{-\mathrm{j} \gamma\left(\left(\frac{-N_{x}}{2}+h_{x}-1\right) \Delta x_{0}\right.\right. \\
& \left.\left.\left(\frac{-N_{y}}{2}+h_{y}-1\right) \Delta y_{0}\right)\right\} \exp \left\{\mathrm{j} \gamma\left(\left(\frac{N_{x}}{2}-1\right) \Delta x_{0},\left(\frac{N_{y}}{2}-1\right) \Delta y_{0}\right)\right\} \\
D\left(h_{x}, h_{y}\right)= & a\left(h_{x}, h_{y}\right) d\left(h_{x}, h_{y}\right)-b\left(h_{x}, h_{y}\right) c\left(h_{x}, h_{y}\right) .
\end{aligned}
$$

Using these variables $\left(h_{x}=2, h_{y}=1\right)$ in Eqs. (25) and (26), we see that

$$
\begin{gathered}
f 1_{0,0}^{*}\left(\left(\frac{-N_{y}}{2}\right) \Delta x_{0},\left(\frac{-N_{y}}{2}\right) \Delta y_{0}\right) f 1_{0,0}\left[\left(\frac{N_{x}}{2}-2\right) \Delta x_{0},\left(\frac{N_{y}}{2}-1\right) \Delta y_{0}\right] \\
=\frac{\operatorname{RHS1}\left(N_{x}-2, N_{y}-1\right) d(2,1)-\operatorname{RHS} 2\left(N_{x}-2, N_{y}-1\right) b(2,1)}{D(2,1)}
\end{gathered}
$$

and

$$
\begin{gathered}
f 1_{0,0}^{*}\left(\left(\frac{-N_{x}}{2}\right) \Delta x_{0},\left(\frac{-N_{y}}{2}\right) \Delta y_{0}\right) f 1_{0,0}\left[\left(\frac{N_{x}}{2}-2\right) \Delta x_{0},\left(\frac{N_{y}}{2}-1\right) \Delta y_{0}\right] \\
=\frac{\operatorname{RHS} 2\left(N_{x}-2, N_{y}-1\right) a(2,1)-\operatorname{RHS1}\left(N_{x}-2, N_{y}-1\right) c(2,1)}{D(2,1)} .
\end{gathered}
$$

Similarly taking $\left(h_{x}=1, h_{y}=2\right)$ in Eqs. (25) and (26) we see that

$$
\begin{gathered}
f 1_{0,0}^{*}\left(\left(\frac{-N_{x}}{2}\right) \Delta x_{0},\left(\frac{-N_{y}}{2}\right) \Delta y_{0}\right) f 1_{0,0}\left[\left(\frac{N_{x}}{2}-1\right) \Delta x_{0},\left(\frac{N_{y}}{2}-2\right) \Delta y_{0}\right] \\
=\frac{\operatorname{RHS} 1\left(N_{x}-1, N_{y}-2\right) d(1,2)-\operatorname{RHS} 2\left(N_{x}-1, N_{y}-2\right) b(1,2)}{D(1,2)}
\end{gathered}
$$

and

$$
\begin{aligned}
f & 1_{0,0}^{*}\left(\left(\frac{-N_{x}}{2}\right) \Delta x_{0},\left(\frac{-N_{y}}{2}\right) \Delta y_{0}\right) f 1_{0,0}\left[\left(\frac{N_{x}}{2}-1\right) \Delta x_{0},\left(\frac{N_{y}}{2}-2\right) \Delta y_{0}\right] \\
& =\frac{\operatorname{RHS} 2\left(N_{x}-1, N_{y}-2\right) a(1,2)-\operatorname{RHS} 1\left(N_{x}-1, N_{y}-2\right) c(1,2)}{D(1,2)} .
\end{aligned}
$$

We can write a general set of expressions for all the remaining values of $h_{x}$ and $h_{y}$; where $1 \leqslant h_{x} \leqslant \frac{N_{x}}{2}+1$ and $1 \leqslant h_{y} \leqslant \frac{N_{y}}{2}+1$

$$
\begin{gathered}
f 1_{0,0}^{*}\left(\left(\frac{-N_{x}}{2}\right) \Delta x_{0},\left(\frac{-N_{y}}{2}\right) \Delta y_{0}\right) f 1_{0,0}\left[\left(\frac{N_{x}}{2}-h_{x}\right) \Delta x_{0},\left(\frac{N_{y}}{2}-h_{y}\right) \Delta y_{0}\right] \\
=\frac{T\left(h_{x}, h_{y}\right) d\left(h_{x}, h_{y}\right)-S\left(h_{x}, h_{y}\right) b\left(h_{x}, h_{y}\right)}{D\left(h_{x}, h_{y}\right)}
\end{gathered}
$$


and

$$
\begin{aligned}
& f 1_{0,0}^{*}\left(\left(\frac{-N_{x}}{2}+h_{x}-1\right) \Delta x_{0},\left(\frac{-N_{y}}{2}+h_{y}-1\right) \Delta y_{0}\right) f 1_{0,0}\left[\left(\frac{N_{x}}{2}-1\right) \Delta x_{0},\left(\frac{N_{y}}{2}-1\right) \Delta y_{0}\right] \\
& \quad=\frac{T\left(h_{x}, h_{y}\right) d\left(h_{x}, h_{y}\right)-S\left(h_{x}, h_{y}\right) b\left(h_{x}, h_{y}\right)}{D\left(h_{x}, h_{y}\right)},
\end{aligned}
$$

where $T\left(h_{x}, h_{y}\right)$ and $S\left(h_{x}, h_{y}\right)$ are defined as

$$
\begin{aligned}
T\left(h_{x}, h_{y}\right)= & \operatorname{RHS1}\left(N_{x}-h_{x}, N_{y}-h_{y}\right) \\
& -\sum_{n_{y}=-\frac{N_{y}}{2}}^{\left(-\frac{N_{y}}{2}+h_{y}-1\right)} \sum_{n_{x}=-\frac{N_{x}}{2}}^{\left(-\frac{N_{x}}{2}+h_{x}-1\right)}\left[f 1_{0,0}^{*}\left(n_{x} \Delta x_{0}, n_{y} \Delta y_{0}\right) f 1_{0,0}\left[\left(n_{x}+N_{x}-h_{x}\right) \Delta x_{0},\left(n_{y}+N_{y}-h_{y}\right) \Delta y_{0}\right]\right. \\
& \left.\times \exp \left\{\mathrm{j} 2 \pi \cot \left(\frac{p_{x} \pi}{2}\right) n_{x}\left(N_{x}-h_{x}\right)\left(\Delta x_{0}\right)^{2}\right\} \exp \left\{\mathrm{j} 2 \pi \cot \left(\frac{p_{y} \pi}{2}\right) n_{y}\left(N_{y}-h_{y}\right)\left(\Delta y_{0}\right)^{2}\right\}\right]
\end{aligned}
$$

and

$$
\begin{aligned}
S\left(h_{x}, h_{y}\right)= & \operatorname{R} H S 2\left(N_{x}-h_{x}, N_{y}-h_{y}\right)-\sum_{n_{y}=-\frac{N_{y}}{2}}^{\left(-\frac{N_{y}}{2}+h_{y}-1\right)} \sum_{n_{x}=-\frac{N_{x}}{2}}^{\left(-\frac{N_{x}}{2}+h_{x}-1\right)}\left[f 1 _ { 0 , 0 } ^ { * } ( n _ { x } \Delta x _ { 0 } , n _ { y } \Delta y _ { 0 } ) f 1 _ { 0 , 0 } \left[\left(n_{x}+N_{x}-h_{x}\right) \Delta x_{0},\right.\right. \\
& \left.\times\left(n_{y}+N_{y}-h_{y}\right) \Delta y_{0}\right] \exp \left\{\mathrm{j} 2 \pi \cot \left(\frac{p_{x} \pi}{2}\right) n_{x}\left(N_{x}-h_{x}\right)\left(\Delta x_{0}\right)^{2}\right\} \\
& \times \exp \left\{\mathrm{j} 2 \pi \cot \left(\frac{p_{y} \pi}{2}\right) n_{y}\left(N_{y}-h_{y}\right)\left(\Delta y_{0}\right)^{2}\right\} \exp \left\{-\mathrm{j} \gamma\left(n_{x} \Delta x_{0}, n_{y} \Delta y_{0}\right)\right\} \\
& \left.\times \exp \left\{\mathrm{j} \gamma\left(\left(n_{x}+N_{x}-h_{x}\right) \Delta x_{0},\left(n_{y}+N_{y}-h_{y}\right) \Delta y_{0}\right)\right\}\right] .
\end{aligned}
$$

In Eqs. (40) and (41) we do not include the summation elements at the following values of $n_{x}$ and $n_{y}$

$$
\left(n_{x}, n_{y}\right)=\left(\frac{N_{x}}{2}, \frac{N_{y}}{2}\right) \quad \text { and } \quad\left(n_{x}, n_{y}\right)=\left(-\frac{N_{x}}{2}+h_{x}-1,-\frac{N_{y}}{2}+h_{y}-1\right) .
$$

We note that the $f 1_{0,0}^{*}\left(n_{x} \Delta x_{0}, n_{y} \Delta y_{0}\right) f 1_{0,0}\left[\left(n_{x}+N_{x}-h_{x}\right) \Delta x_{0},\left(n_{y}+N_{y}-h_{y}\right) \Delta y_{0}\right]$ terms in the Eqs. (40) and (41) can be determined by multiplying the previously calculated term $f 1_{0,0}^{*}\left(\left(\frac{-N_{x}}{2}\right) \Delta x_{0},\left(\frac{-N_{x}}{2}\right) \Delta y_{0}\right) f 1_{0,0}$ $\left[\left(\frac{-N_{x}}{2}-h_{x}\right) \Delta x_{0},\left(\frac{-N_{y}}{2}-h_{y}\right) \Delta y_{0}\right]$ given by Eq. (38) and

$$
f 1_{0,0}^{*}\left(\left(\frac{-N_{x}}{2}+h_{x}-1\right) \Delta x_{0},\left(\frac{-N_{y}}{2}+h_{y}-1\right) \Delta y_{0}\right) f 1_{0,0}\left[\left(\frac{-N_{x}}{2}-1\right) \Delta x_{0},\left(\frac{-N_{y}}{2}-1\right) \Delta y_{0}\right]
$$

given by Eq. (39) and dividing the result of the multiplication by the value

$$
f 1_{0,0}^{*}\left(\left(\frac{-N_{x}}{2}\right) \Delta x_{0},\left(\frac{-N_{y}}{2}\right) \Delta y_{0}\right) f 1_{0,0}\left[\left(\frac{-N_{x}}{2}-1\right) \Delta x_{0},\left(\frac{-N_{y}}{2}-1\right) \Delta y_{0}\right]
$$

found by Eq. (27).

It is shown in Appendix B that it is extremely unlikely that $D\left(h_{\mathrm{x}}, h_{\mathrm{y}}\right)$ is ever zero. When we reach $h_{\mathrm{x} 0}=\left(N_{\mathrm{x}} / 2+1\right)$ and $h_{\mathrm{y} 0}=\left(N_{\mathrm{y}} / 2+1\right)$ we note that

$$
\begin{aligned}
& f_{0,0}\left[\left(\frac{N_{x}}{2}-\left(h_{x 0}-1\right)\right) \Delta x_{0},\left(\frac{-N_{y}}{2}-\left(h_{y 0}-1\right)\right) \Delta y_{0}\right] \\
& \quad=f_{0,0}\left(\left(\frac{-N_{x}}{2}+h_{x 0}-1\right) \Delta x_{0},\left(\frac{-N_{x}}{2}+h_{y 0}-1\right) \Delta y_{0}\right)
\end{aligned}
$$

the above expression is equivalent to $f_{0,0}(0,0)=f_{0,0}(0,0)$. 
These equations allow us to determine $f_{0,0}(0,0)$ completely since

$$
\begin{aligned}
& \left\{f 1_{0,0}^{*}\left(-\frac{N_{x}}{2} \Delta x_{0}, \frac{-N_{y}}{2} \Delta y_{0}\right) f 1_{0,0}\left[\left(\frac{N_{x}}{2}-\left(h_{x 0}-1\right)\right) \Delta x_{0},\left(\frac{N_{y}}{2}-\left(h_{y 0}-1\right)\right) \Delta y_{0}\right] f 1_{0,0}^{*}\right. \\
& \left.\quad \times\left[\left(\frac{-N_{x}}{2}+h_{x 0}-1\right) \Delta x_{0},\left(\frac{-N_{y}}{2}+h_{y 0}-1\right) \Delta y_{0}\right] f 1_{0,0}\left[\left(\frac{N_{x}}{2}-1\right) \Delta x_{0},\left(\frac{N_{y}}{2}-1\right) \Delta y_{0}\right]\right\} \\
& \quad /\left\{\operatorname{RHS1}\left(N_{x}-1, N_{y}-1\right) \exp \left[\mathrm{j} \pi \cot \left(\frac{p_{x} \pi}{2}\right) N_{x}\left(N_{x}-h_{x}\right)\left(\Delta x_{0}\right)^{2}\right]\right. \\
& \left.\quad \times \exp \left[\mathrm{j} \pi \cot \left(\frac{p_{y} \pi}{2}\right) N_{y}\left(N_{y}-h_{y}\right)\left(\Delta y_{0}\right)^{2}\right]\right\}=\left|f 1_{0,0}(0,0)\right|^{2} .
\end{aligned}
$$

Once we have this value we can choose an arbitrary phase for it and dividing it into Eqs. (38) and (39) for the appropriate values of $h_{x}$ and $h_{y}$, determine values for $f 1_{0,0}\left(\left(\frac{-N_{x}}{2}\right) \Delta x_{0},\left(\frac{-N_{y}}{2}\right) \Delta y_{0}\right)$ and $f 1_{0,0}\left[\left(\frac{N_{x}}{2}-1\right)\right.$ $\left.\Delta x_{0},\left(\frac{N_{y}}{2}-1\right) \Delta y_{0}\right]$. To find all other values of $f 1$ can now divide these two values into the products provided by Eqs. (38) and (39) for all values of $h_{x}$ and $h_{y}$.

Finally, to recover our original signal, we multiply by $\exp \left\{-\mathrm{j} \phi\left(l_{x} \Delta x_{0}, l_{y} \Delta y_{0}\right)\right\}$, the complex conjugate of the first random phase key used to encrypt our signal:

$$
g_{0,0}\left(l_{x} \Delta x_{0}, l_{y} \Delta y_{0}\right)=f 1_{0,0}\left(l_{x} \Delta x_{0}, l_{y} \Delta y_{0}\right) \exp \left\{-\mathrm{j} \phi\left(l_{x} \Delta x_{0}, l_{y} \Delta y_{0}\right)\right\} .
$$

The 1D phase retrieval algorithm outlined in [22] cannot be simply extended to 2D because $D\left(h_{x}\right)$ defined in [22] is guaranteed under specific constraints never to equal zero for all the relevant values of $h_{x}$ but extending the algorithm to more one dimension causes $D\left(h_{x}, h_{y}\right)$ to have zero values for certain values of $\left(h_{x}, h_{y}\right)$. This problem is overcome in our technique by the phase difference introduced by us in Eq. (22). This is discussed in more detail in Appendix B. Indeed, this algorithm could be used purely for phase retrieval in an analogous way to that described in [22]. In this case, we would only require one phase plane, which would not have to be random.

As stated above, in [22], it was necessary to impose a relationship upon the orders $p_{x}$ and $q_{x}$ in order to ensure $D\left(h_{x}\right)$ is never equal to zero. In our algorithm, there is no restriction on the FRT orders we use (see Appendix B). However, the algorithm is more prone to errors occurring in the recursive procedure if the orders $p_{x}$ and $q_{x}$ and also $p_{y}$ and $q_{y}$ are close to each other.

\section{Results}

We present results of the simulations of four encryption processes for the same image and different sampling densities (image pixel sizes). For simplicity only results for the encryption of an amplitude signal are presented even though, as was pointed out earlier, it is possible to encrypt a second image on the phase of this input signal. The image sizes used were $16 \times 16,32 \times 32,64 \times 64$ and $128 \times 128$. In all the simulations of encryption presented here $\left(p_{x}=0.5, p_{y}=0.5\right)$, and $\left(q_{x}=1.5, q_{y}=1.5\right)$. The decryption process was found to be extremely sensitive to small computational errors. For this reason, it was necessary to quantise the input data and then, during the decryption recursive loop, to use our a priori knowledge regarding the quantisation levels employed to remove any errors in the values. The input image was also normalised before the encryption procedure so that the maximum intensity value was equal to 1 .

In the proceeding sections, the mean square error (MSE) is used as a measure of the level of encryption of encrypted and incorrectly decrypted images. We note that it refers to the average square of the difference between the pixel values of the correct image and the image being analysed. We also note that before MSE is calculated the correct image and the analysed image are brought back to their initial quantisation level where we deal with pixel values between 0 and 256 corresponding to the familiar grayscale representation. 
The MSE of incorrectly decrypted images is unusually large because the decryption algorithm returns values that far exceed 255 in these cases. Fig. 2 shows results for the $32 \times 32$ case. The input image is shown in Fig. 2(a). The encrypted image is shown in Fig. 2(b), which displays the amplitude of a signal, whose real and imaginary parts are given by the two intensities obtained from the encryption procedure. The MSE of

(a)

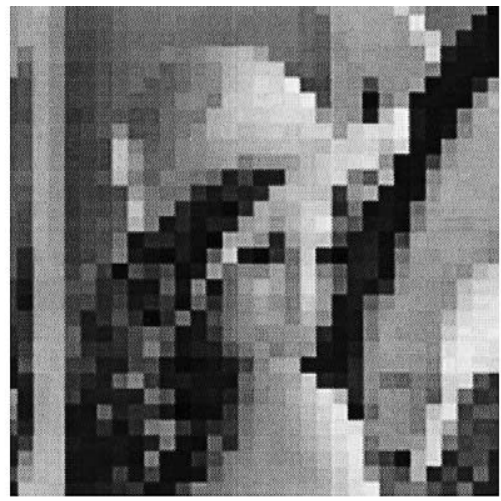

(c)

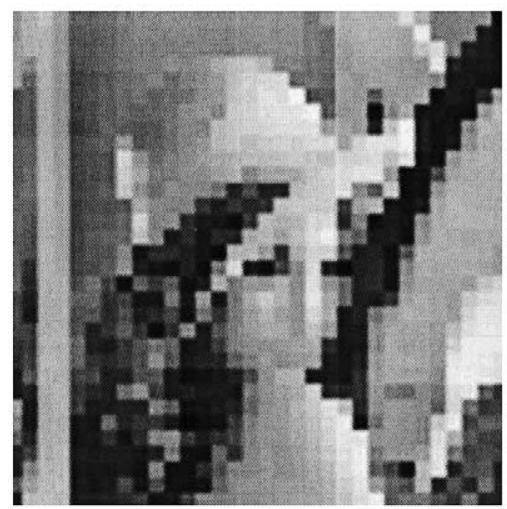

(e)

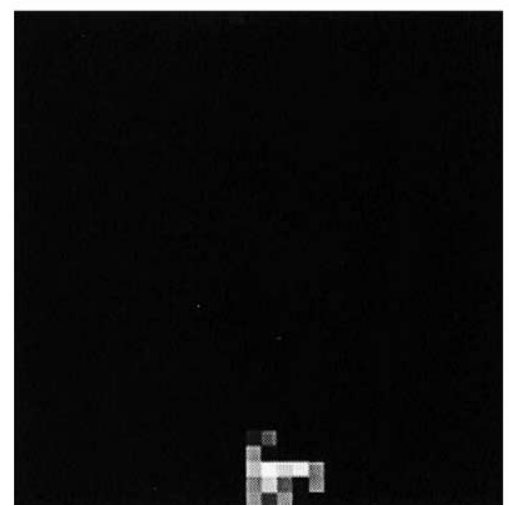

(b)

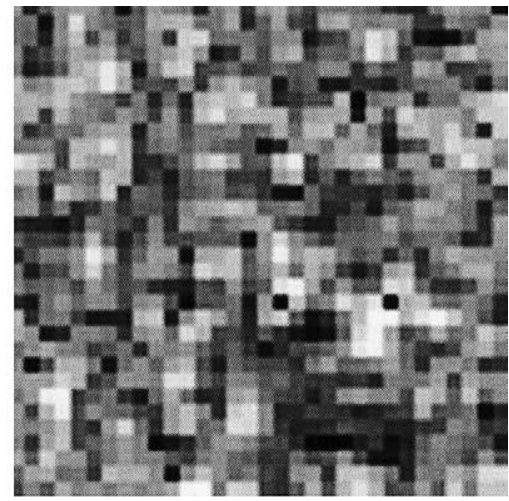

(d)

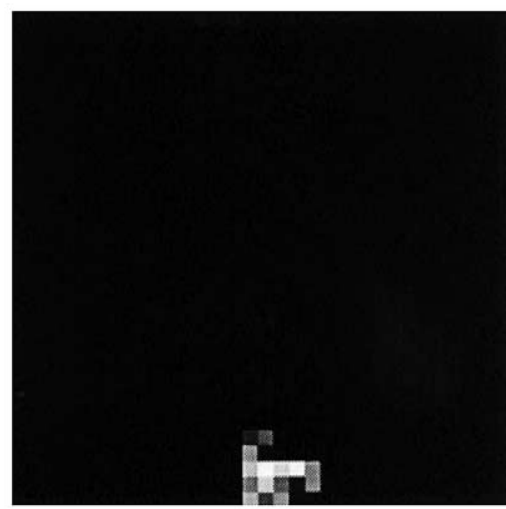

(f)

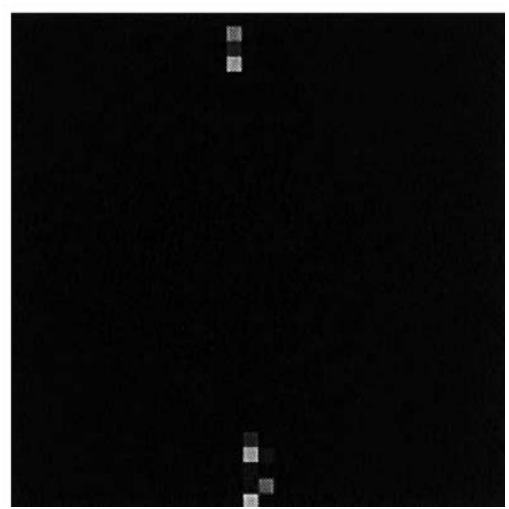

Fig. 2. Encryption/decryption results for $32 \times 32$ image: (a) input image; (b) encrypted image; (c) decrypted image; (d) decrypted using an incorrect value of $p_{x}$, out by $1 \times 10^{-5}$; (e) decrypted using an incorrect value of $q_{x}$, out by $1 \times 10^{-5}$; (f) decrypted using an incorrect phase key. 
this image was calculated to be 17394.69. A correctly decrypted image is shown in Fig. 2(c) with a MSE of 0.40. In Fig. 2(d), we show the result of decrypting with $p_{x}=0.50001$, i.e., an error of $1 \times 10^{-5}$ in the FRT order in the $x$-direction. The resulting image has a MSE of $1.46 \times 10^{44}$. In Fig. 2(e), we show the result of decrypting with $p_{y}=0.50001$. The resulting image has a MSE of $1.37 \times 10^{43}$. In Fig. 2(f), we show the result of decrypting the signal using all the correct fractional order keys but a completely incorrect phase key. The MSE of this image was calculated to be $1.51 \times 10^{75}$.

Figs. 3 and 4 correspond to the $32 \times 32$ case. Both show how deviations from the correct values for $p_{x}$ and $q_{x}$ effect the MSE of the resulting decrypted image. Fig. 3 shows this variation for very small deviations in the orders, in step sizes of $1.0 \times 10^{-6}$ while Fig. 4 is the same case for a wider range of deviations. It can be seen that symmetry exists in the curves for increases in $p_{x}$ and decreases in $q_{x}$ and vice versa.

Similarly, Figs. 5 and 6 both show how deviations from the correct values for $p_{x}$ and $q_{x}$ affect the MSE of the resulting decrypted image for the $64 \times 64$ case. Fig. 5 shows this variation for very small deviations in the orders, with step sizes of $1.0 \times 10^{-9}$ while Fig. 6 extends this range to larger deviations. Again there is symmetry for increases in $p_{x}$ and decreases in $q_{x}$ and vice versa. The piecewise linearity of the pattern shown in Fig. 5 is due to the quantisation of the data values.

Attempts were made to carry out the same analysis for the $128 \times 128$ case. While it was possible to carry out both encryption and correct decryption it was not possible to calculate the MSE of incorrectly decrypted images because the data generated in the decryption process exceeded values that could be processed by the simulation software employed using standard means.

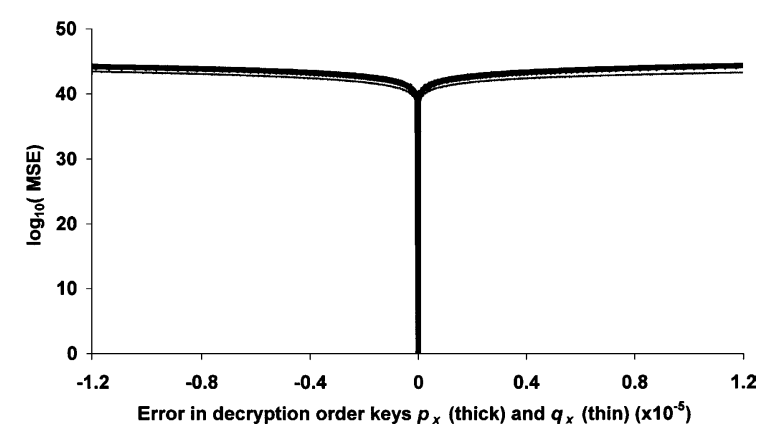

Fig. 3. Small error in decryption order keys used versus the resultant MSE of the decrypted $32 \times 32$ image.

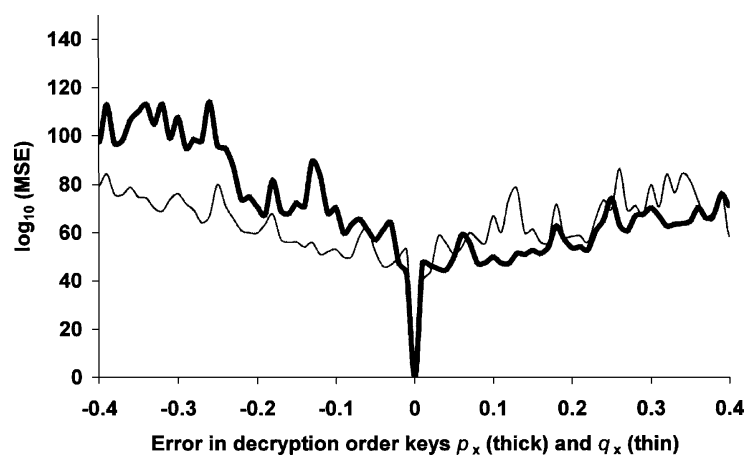

Fig. 4. Error in decryption order keys used versus the resultant MSE of the decrypted $32 \times 32$ image. 


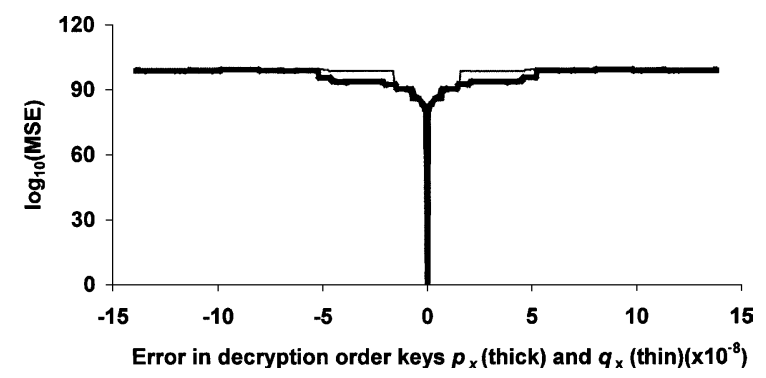

Fig. 5. Small error in decryption order keys used versus the resultant MSE of the decrypted $64 \times 64$ image.

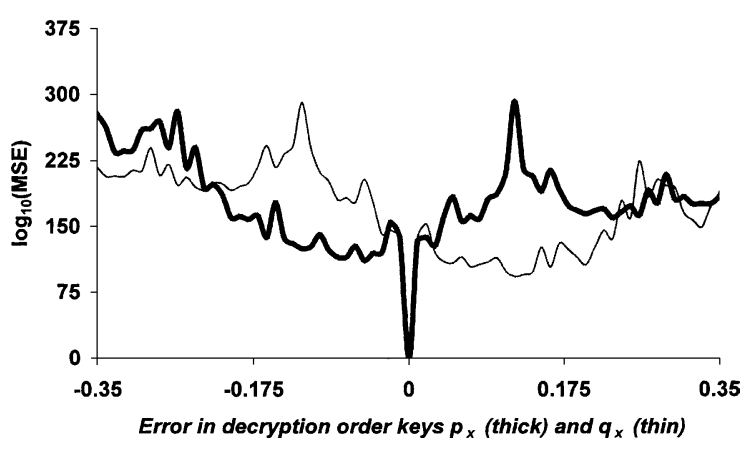

Fig. 6. Error in decryption order keys used versus the resultant MSE of the decrypted $64 \times 64$ image.

As we analysed the results for the different sized images a trend emerged. The sensitivity of the fractional order keys increased and the MSE of incorrectly decrypted images also increased with the size of the image being encrypted. The reason for this is that larger images require a correspondingly larger recursive procedure. Errors propagate and accumulate through the algorithm the greater the number of iterations required. It is also worth noting that the last two values calculated by this recursive procedure are necessary in order to determine $f_{0,0}(0,0)$, which must be accurately determined in order to find all the other data values. This implies that if an error propagates from any point in the recursive procedure, then it will affect all of the pixel value estimates.

For the sake of comparison we present the same analysis for two other encryption schemes, which incorporate the FRT. The first of these is based on the procedure presented in [13] which consists of taking an input (image) wave field and adding a random phase, carrying out an FRT of arbitrary orders and applying a second random phase and finally carrying out a second FRT of another set of arbitrary orders. Decryption is the exact inverse of the encryption procedure, using the negatives of the FRT orders originally used and the conjugates of the phase keys. We can examine sensitivity of the two FRT order keys when decrypting the encoded image in Fig. 7. These should be compared with the results in Figs. 3-6. The encryption procedure presented in this paper shows a considerable increase in sensitivity to the FRT order keys used in terms of the size of the MSE of the incorrectly decrypted images. The second algorithm we compare with our results is that presented in [12]. This method uses three FRT operations and instead of using phase keys in these arbitrary FRT domains, a random juxtaposing of sections of the image is used instead. The sensitivity of the three FRT order keys is shown in Fig. 8. Again these can be compared with the results in Figs. 3-6. We can see that the encryption procedure presented in this paper again demonstrates a considerable increase in sensitivity of the FRT keys used as indicated by the MSE of the incorrectly decrypted images. 


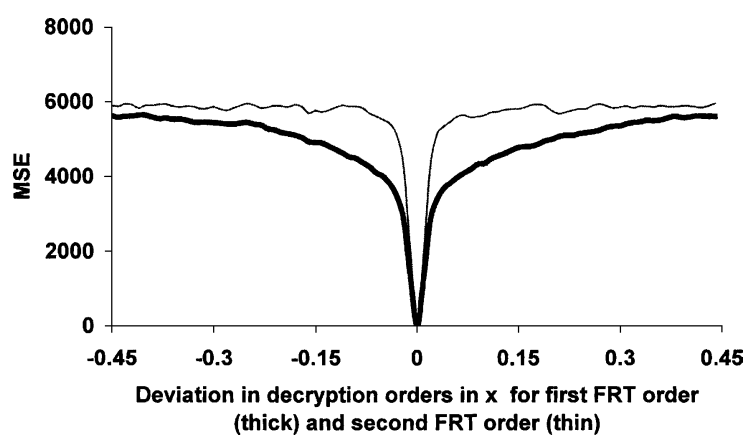

Fig. 7. Error in decryption order keys for the method presented in [13] versus the resultant MSE of the decrypted images.

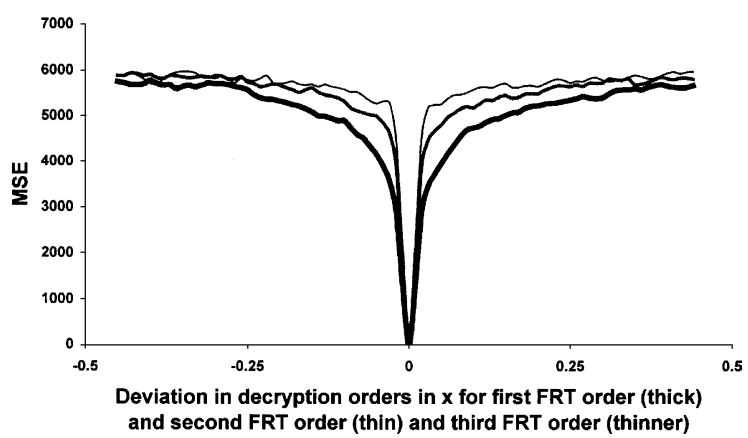

Fig. 8. Error in decryption order keys for the method presented in [12] versus the resultant MSE of the decrypted images.

It is also possible to compare the number of recursive iterations for the different sized images. The recursive part of the decryption process requires the following number of iterations

$$
\text { Number of recursive iterations }=\sum_{h_{y}=1}^{\left(N_{y}-1\right)} \sum_{h_{x}=1}^{\left(\frac{N_{x}}{2}-1\right)} \sum_{l_{y}=-\frac{N_{y}}{2}}^{\left(-\frac{N_{y}}{2}+h_{y}-1\right)} \sum_{l_{y}=-\frac{N_{x}}{2}}^{\left(-\frac{N_{x}}{2}+h_{x}-1\right)} 1 .
$$

For the $16 \times 16\left(N_{x}=N_{y}=16\right)$ case the number of iterations is equal to 6120 , for the $32 \times 32$ case the number of iterations is equal to 80784 , for the $64 \times 64$ case the number of iterations is 1166880 and finally for the $128 \times 128$ case the number of iterations required is $17,709,120$.

\section{Conclusions}

In this paper, we have discussed the definition and some of the properties of the FRT and one of its discrete forms. Using the correlation property we have developed a 2D encryption procedure by extending the 1D phase retrieval procedure presented in [22]. Simulations show that the encryption keys are far more sensitive than those of similar FRT-based encryption schemes. They are so sensitive in fact that the encryption scheme could only be implemented with great difficulty using traditional optical methods, since we would have to know the parameters of the system, namely the orders of the optical FRT transform, extremely accurately. Furthermore, the larger the input number of pixels the more accurately we need to know these parameters. The results presented show the proposed technique to be a highly effective method for the digital encryption of multidimensional data. 


\section{Acknowledgements}

The authors would like to acknowledge the support of Enterprise Ireland through the Research Innovation Fund.

\section{Appendix A. Correlation property proof}

If we expand the left hand side of Eq. (9) using the inverse relation to determine $f_{0}$ we get

$$
\begin{aligned}
& \sum_{l_{y}=-\frac{N_{y}}{2}}^{\frac{N_{y}}{2}-1} \sum_{l_{x}=-\frac{N_{x}}{2}}^{\frac{N_{x}}{2}-1}\left[f_{0,0}^{*}\left(l_{x} \Delta x_{0}, l_{y} \Delta y_{0}\right) f_{0,0}\left[\left(l_{x}+k_{x}\right) \Delta x_{0},\left(l_{y}+k_{y}\right) \Delta y_{0}\right]\right. \\
& \left.\times \exp \left\{\mathrm{j} 2 \pi \cot \left(\frac{p_{x} \pi}{2}\right) l_{x} k_{x}\left(\Delta x_{0}\right)^{2}\right\} \exp \left\{\mathrm{j} 2 \pi \cot \left(\frac{p_{y} \pi}{2}\right) l_{y} k_{y}\left(\Delta y_{0}\right)^{2}\right\}\right] \\
& =\sum_{l_{y}=-\frac{N_{y}}{2}}^{\frac{N_{y}}{2}-1} \sum_{l_{x}=-\frac{N_{x}}{2}}^{\frac{N_{x}}{2}-1}\left[\left[F^{-p_{x},-p_{y}}\left\{f_{p_{x}, p_{y}}\left(m_{x} \Delta x_{p_{x}}, m_{y} \Delta y_{p_{y}}\right)\right\}\left(l_{x} \Delta x_{0}, l_{y} \Delta y_{0}\right)\right]^{*}\right. \\
& \times\left[F^{-P x,-P y}\left\{f_{p_{x}, p_{y}}\left(m_{x} \Delta x_{p_{x}}, m_{y} \Delta y_{p_{y}}\right)\right\}\left(\left(l_{x}+k_{x}\right) \Delta x_{0},\left(l_{y}+k_{y}\right) \Delta y_{0}\right)\right] \\
& \left.\times \exp \left\{\mathrm{j} 2 \pi \cot \left(\frac{p_{x} \pi}{2}\right) l_{x} k_{x}\left(\Delta x_{0}\right)^{2}\right\} \exp \left\{\mathrm{j} 2 \pi \cot \left(\frac{p_{y} \pi}{2}\right) l_{y} k_{y}\left(\Delta y_{0}\right)^{2}\right\}\right] \\
& =\sum_{l_{y}=-\frac{N_{y}}{2}}^{\frac{N_{y}}{2}-1} \sum_{l_{x}=-\frac{N_{x}}{2}}^{\frac{N_{x}}{2}-1}\left\{\left[A_{-p_{x}} A_{-p_{y}} \Delta x_{p_{x}} \Delta y_{p_{y}} \sum_{m_{y}=-\frac{N_{y}}{2}}^{\frac{N_{y}}{2}-1} \sum_{m_{x}=-\frac{N_{x}}{2}}^{\frac{N_{x}}{2}-1} f_{p_{x}, p_{y}}\left(m_{x} \Delta x_{p_{x}}, m_{y} \Delta y_{p_{y}}\right)\right.\right. \\
& \times \exp \left\{-\mathrm{j} \pi \cot \left(\frac{p_{x} \pi}{2}\right)\left[\left(l_{x} \Delta x_{0}\right)^{2}+\left(m_{x} \Delta x_{p_{x}}\right)^{2}\right]\right\} \exp \left\{\mathrm{j} 2 \pi \frac{l_{x} m_{x}}{N_{x}}\right\} \\
& \left.\times \exp \left\{-\mathrm{j} \pi \cot \left(\frac{p_{y} \pi}{2}\right)\left[\left(l_{y} \Delta y_{0}\right)^{2}+\left(m_{y} \Delta y_{p_{y}}\right)^{2}\right]\right\} \exp \left\{\mathrm{j} 2 \pi \frac{l_{y} m_{y}}{N_{y}}\right\}\right]^{*} \\
& \times\left[A_{-p_{x}} A_{-p_{y}} \Delta x_{p_{x}} \Delta y_{p_{y}} \sum_{m_{y}=\frac{N_{y}}{2}}^{\frac{N_{y}}{2}-1} \sum_{m_{x}=\frac{-N_{x}}{2}}^{\frac{N_{x}}{2}-1} f_{p_{x}, p_{y}}\left(m_{x} \Delta x_{p_{x}}, m_{y} \Delta y_{p_{y}}\right)\right. \\
& \times \exp \left\{-\mathrm{j} \pi \cot \left(\frac{p_{x} \pi}{2}\right)\left[\left(\left(l_{x}+k_{x}\right) \Delta x_{0}\right)^{2}+\left(m_{x} \Delta x_{p_{x}}\right)^{2}\right]\right\} \exp \left\{\mathrm{j} 2 \pi \frac{\left(l_{x}+k_{x}\right) m_{x}}{N_{x}}\right\} \\
& \left.\times \exp \left\{-\mathrm{j} \pi \cot \left(\frac{p_{y} \pi}{2}\right)\left[\left(\left(l_{y}+k_{y}\right) \Delta y_{0}\right)^{2}+\left(m_{y} \Delta y_{p_{y}}\right)^{2}\right]\right\} \exp \left\{\mathrm{j} 2 \pi \frac{\left(l_{y}+k_{y}\right) m_{y}}{N_{y}}\right\}\right] \\
& \left.\times \exp \left\{\mathrm{j} 2 \pi \cot \left(\frac{p_{x} \pi}{2}\right) l_{x} k_{x}\left(\Delta x_{0}\right)^{2}\right\} \exp \left\{\mathrm{j} 2 \pi \cot \left(\frac{p_{y} \pi}{2}\right) l_{y} k_{y}\left(\Delta y_{0}\right)^{2}\right\}\right\}
\end{aligned}
$$




$$
\begin{aligned}
& =\left|A_{p_{x}}\right|^{2}\left|A_{p_{y}}\right|^{2} \Delta x_{p_{x}}^{2} \Delta y_{p_{y}}^{2} \sum_{l_{y}=-\frac{N_{y}}{2}}^{\frac{N_{y}}{2}-1} \sum_{l_{x}=-\frac{N_{x}}{2}}^{\frac{N_{x}}{2}-1}\left\{\left[\sum_{m_{y}=\frac{N_{y}}{2}}^{\frac{N_{y}}{2}-1} \sum_{m_{x}=\frac{-N_{x}}{2}}^{\frac{N_{x}}{2}-1} f_{p_{x}, p_{y}}\left(m_{x} \Delta x_{p_{x}}, m_{y} \Delta y_{p_{y}}\right)\right.\right. \\
& \times \exp \left\{-\mathrm{j} \pi \cot \left(\frac{p_{x} \pi}{2}\right)\left[\left(l_{x} \Delta x_{0}\right)^{2}+\left(m_{x} \Delta x_{p_{x}}\right)^{2}\right]\right\} \exp \left\{\mathrm{j} 2 \pi \frac{l_{x} m_{x}}{N_{x}}\right\} \\
& \left.\times \exp \left\{-\mathrm{j} \pi \cot \left(\frac{p_{y} \pi}{2}\right)\left[\left(l_{y} \Delta y_{0}\right)^{2}+\left(m_{y} \Delta y_{p_{y}}\right)^{2}\right]\right\} \exp \left\{\mathrm{j} 2 \pi \frac{l_{y} m_{y}}{N_{y}}\right\}\right]^{*} \\
& \times\left[\sum_{m_{y}=\frac{-N_{y}}{2}}^{\frac{N_{y}}{2}-1} \sum_{m_{x}=\frac{-N_{x}}{2}}^{\frac{N_{x}}{2}-1} f_{p_{x}, p_{y}}\left(m_{x} \Delta x_{p_{x}}, m_{y} \Delta y_{p_{y}}\right) \exp \left\{-\mathrm{j} \pi \cot \left(\frac{p_{x} \pi}{2}\right)\left[\left(l_{x} \Delta x_{0}\right)^{2}+\left(m_{x} \Delta x_{p_{x}}\right)^{2}\right]\right\} \exp \left\{\mathrm{j} 2 \pi \frac{l_{x} m_{x}}{N_{x}}\right\}\right. \\
& \times \exp \left\{\mathrm{j} 2 \pi \frac{m_{x} k_{x}}{N_{x}}\right\} \exp \left\{-\mathrm{j} \pi \cot \left(\frac{p_{y} \pi}{2}\right)\left[\left(l_{y} \Delta y_{0}\right)^{2}+\left(m_{y} \Delta y_{p_{y}}\right)^{2}\right]\right\} \exp \left\{\mathrm{j} 2 \pi \frac{l_{y} m_{y}}{N_{y}}\right\} \\
& \left.\left.\quad \times \exp \left\{\mathrm{j} 2 \pi \frac{m_{y} k_{y}}{N_{y}}\right\}\right] \exp \left\{\mathrm{j} 2 \pi \cot \left(\frac{p_{x} \pi}{2}\right) l_{x} k_{x}\left(\Delta x_{0}\right)^{2}\right\} \exp \left\{\mathrm{j} 2 \pi \cot \left(\frac{p_{y} \pi}{2}\right) l_{y} k_{y}\left(\Delta y_{0}\right)^{2}\right\}\right\} .
\end{aligned}
$$

Since the inner two summations are over an identical range we can place them within one summation over a single variable.

$$
\begin{aligned}
= & \left|A_{p_{x}}\right|^{2}\left|A_{p_{y}}\right|^{2} \Delta x_{p_{x}}^{2} \Delta y_{p_{y}}^{2} \sum_{l_{y}=-\frac{N_{y}}{2}}^{\frac{N_{y}}{2}-1} \sum_{l_{x}=-\frac{N_{x}}{2}}^{\frac{N_{x}}{2}-1}\left\{\left[\sum_{m_{y}=\frac{-N_{y}}{2}}^{\frac{N_{y}}{2}-1} \sum_{m_{x}=\frac{N_{x}}{2}}^{\frac{N_{x}}{2}-1}\left|f_{p_{x}, p_{y}}\left(m_{x} \Delta x_{p_{x}}, m_{y} \Delta y_{p_{y}}\right)\right|^{2} \exp \left\{\mathrm{j} 2 \pi \frac{k_{x} m_{x}}{N_{x}}\right\} e\left\{\mathrm{j} 2 \pi \frac{k_{x} m_{x}}{N_{x}}\right\}\right]\right. \\
& \left.\times \exp \left\{\mathrm{j} \pi \cot \left(\frac{p_{x} \pi}{2}\right) k_{x}^{2}\left(\Delta x_{0}\right)^{2}\right\} \exp \left\{\mathrm{j} \pi \cot \left(\frac{p_{y} \pi}{2}\right) k_{y}^{2}\left(\Delta y_{0}\right)^{2}\right\}\right\} \\
= & \left|A_{p_{x}}\right|^{2}\left|A_{p_{y}}\right|^{2} \Delta x_{p_{x}}^{2} \Delta y_{p_{y}}^{2} N_{x} N_{y} \exp \left\{\mathrm{j} \pi \cot \left(\frac{p_{x} \pi}{2}\right) k_{x}^{2}\left(\Delta x_{0}\right)^{2}\right\} \exp \left\{\mathrm{j} \pi \cot \left(\frac{p_{y} \pi}{2}\right) k_{y}^{2}\left(\Delta y_{0}\right)^{2}\right\} \\
& \times \sum_{m_{y}=\frac{N_{y}}{2}}^{\frac{N_{y}}{2}-1} \sum_{m_{x}=\frac{-N_{x}}{2}}^{\frac{N_{x}}{2}-1}\left|f_{p_{x}, p_{y}}\left(m_{x} \Delta x_{p_{x}}, m_{y} \Delta y_{p_{y}}\right)\right|^{2} \exp \left\{\mathrm{j} 2 \pi \frac{k_{x} m_{x}}{N_{x}}\right\} \exp \left\{\mathrm{j} 2 \pi \frac{k_{x} m_{x}}{N_{x}}\right\} \\
= & \frac{\left|\sin \left(\frac{p_{x} \pi}{2}\right)\right|}{N_{x} \Delta x_{0}^{2}} \frac{\left|\sin \left(\frac{p_{y} \pi}{2}\right)\right|}{N_{y} \Delta y_{0}^{2}} \exp \left\{\mathrm{j} \pi \cot \left(\frac{p_{x} \pi}{2}\right) k_{x}^{2}\left(\Delta x_{0}\right)^{2}\right\} \exp \left\{\mathrm{j} \pi \cot \left(\frac{p_{y} \pi}{2}\right) k_{y}^{2}\left(\Delta y_{0}\right)^{2}\right\} \\
& \times \sum_{m_{y}=\frac{N_{y}}{2}}^{\frac{N_{y}}{2}-1} \sum_{m_{x}=\frac{-N_{x}}{2}}^{\frac{N_{x}}{2}-1}\left|f_{p_{x}, p_{y}}\left(m_{x} \Delta x_{p_{x}}, m_{y} \Delta y_{p_{y}}\right)\right|^{2} \exp \left\{\mathrm{j} 2 \pi \frac{k_{x} m_{x}}{N_{x}}\right\} \exp \left\{\mathrm{j} 2 \pi \frac{k_{y} m_{y}}{N_{y}}\right\} .
\end{aligned}
$$




\section{Appendix B. Why D is unlikely to equal zero}

As indicated in the text it is important that the denominator variable appearing in Eq. (33) is not zero. Here we examine the behavior of this variable.

$$
\begin{aligned}
& D\left(h_{x}, h_{y}\right)=a\left(h_{x}, h_{y}\right) d\left(h_{x}, h_{y}\right)-b\left(h_{x}, h_{y}\right) c\left(h_{x}, h_{y}\right) \\
& \times \exp \left\{-\mathrm{j} \pi \cot \left(\frac{p_{x} \pi}{2}\right) N_{x}\left(N_{x}-h_{x}\right)\left(\Delta x_{0}\right)^{2}\right\} \exp \left\{-\mathrm{j} \pi \cot \left(\frac{p_{y} \pi}{2}\right) N_{y}\left(N_{y}-h_{y}\right)\left(\Delta y_{0}\right)^{2}\right\} \\
& \times \exp \left\{-\mathrm{j} \pi \cot \left(\frac{q_{x} \pi}{2}\right)\left(N_{x}-2 h_{x}+2\right)\left(N_{x}-h_{x}\right)\left(\Delta x_{0}\right)^{2}\right\} \\
& \times \exp \left\{-\mathrm{j} \pi \cot \left(\frac{q_{y} \pi}{2}\right)\left(N_{y}-2 h_{y}+2\right)\left(N_{y}-h_{y}\right)\left(\Delta y_{0}\right)^{2}\right\} \\
& \times \exp \left\{-\mathrm{j} \gamma\left(\left(\frac{-N_{x}}{2}+h_{x}-1\right) \Delta x_{0},\left(\frac{-N_{y}}{2}+h_{y}-1\right) \Delta y_{0}\right)\right\} \\
& \times \exp \left\{\mathrm{j} \gamma\left(\left(\frac{N_{x}}{2}+h_{x}-1\right) \Delta x_{0},\left(\frac{N_{y}}{2}+h_{y}-1\right) \Delta y_{0}\right)\right\} \\
& -\exp \left\{-\mathrm{j} \pi \cot \left(\frac{p_{x} \pi}{2}\right)\left(N_{x}-2 h_{x}+2\right)\left(N_{x}-h_{x}\right)\left(\Delta x_{0}\right)^{2}\right\} \\
& \times \exp \left\{-\mathrm{j} \pi \cot \left(\frac{p_{y} \pi}{2}\right)\left(N_{y}-2 h_{y}+2\right)\left(N_{y}-h_{y}\right)\left(\Delta y_{0}\right)^{2}\right\} \exp \left\{-\mathrm{j} \pi \cot \left(\frac{q_{x} \pi}{2}\right) N_{x}\left(N_{x}-h_{x}\right)\left(\Delta x_{0}\right)^{2}\right\} \\
& \times \exp \left\{-\mathrm{j} \pi \cot \left(\frac{q_{y} \pi}{2}\right) N_{y}\left(N_{y}-h_{y}\right)\left(\Delta y_{0}\right)^{2}\right\} \exp \left\{-\mathrm{j} \pi \gamma\left(\left(\frac{-N_{x}}{2}\right) \Delta x_{0},\left(\frac{-N_{y}}{2}\right) \Delta y_{0}\right)\right\} \\
& \times \exp \left\{-\mathrm{j} \pi \gamma\left(\left(\frac{N_{x}}{2}\right) \Delta x_{0},\left(\frac{N_{y}}{2}\right) \Delta y_{0}\right)\right\} \\
& =\exp \left\{-\mathrm{j} \pi \cot \left(\frac{p_{x} \pi}{2}\right) N_{x}\left(N_{x}-h_{x}\right)\left(\Delta x_{0}\right)^{2}\right\} \exp \left\{-\mathrm{j} \pi \cot \left(\frac{p_{y} \pi}{2}\right) N_{y}\left(N_{y}-h_{y}\right)\left(\Delta y_{0}\right)^{2}\right\} \\
& \times \exp \left\{-\mathrm{j} \pi \cot \left(\frac{q_{x} \pi}{2}\right)\left(N_{x}-2 h_{x}+2\right)\left(N_{x}-h_{x}\right)\left(\Delta x_{0}\right)^{2}\right\} \\
& \times \exp \left\{-\mathrm{j} \pi \cot \left(\frac{q_{y} \pi}{2}\right)\left(N_{y}-2 h_{y}+2\right)\left(N_{y}-h_{y}\right)\left(\Delta y_{0}\right)^{2}\right\} \\
& \times \exp \left\{-\mathrm{j} \gamma\left(\left(\frac{-N_{x}}{2}+h_{x}-1\right) \Delta x_{0},\left(\frac{-N_{y}}{2}+h_{y}-1\right) \Delta y_{0}\right)\right\} \\
& \times \exp \left\{\mathrm{j} \gamma\left(\left(\frac{N_{x}}{2}-1\right) \Delta x_{0},\left(\frac{N_{y}}{2}-1\right) \Delta y_{0}\right)\right\} \\
& \times\left[1-\exp \left\{-\mathrm{j} \pi\left(\cot \left(\frac{p_{x} \pi}{2}\right)-\cot \left(\frac{q_{x} \pi}{2}\right)\right)\left(N_{x}-2 h_{x}+2\right)\left(N_{x}-h_{x}\right)\left(\Delta x_{0}\right)^{2}\right\}\right. \\
& \times \exp \left\{-\mathrm{j} \pi\left(\cot \left(\frac{p_{y} \pi}{2}\right)-\cot \left(\frac{q_{y} \pi}{2}\right)\right)\left(N_{y}-2 h_{y}+2\right)\left(N_{y}-h_{y}\right)\left(\Delta y_{0}\right)^{2}\right\} \\
& \times \exp \left\{-\mathrm{j} \pi\left(\cot \left(\frac{p_{x} \pi}{2}\right)-\cot \left(\frac{q_{x} \pi}{2}\right)\right) N_{x}\left(N_{x}-h_{x}\right)\left(\Delta x_{0}\right)^{2}\right\} \\
& \times \exp \left\{-\mathrm{j} \pi\left(\cot \left(\frac{p_{y} \pi}{2}\right)-\cot \left(\frac{q_{y} \pi}{2}\right)\right) N_{y}\left(N_{y}-h_{y}\right)\left(\Delta y_{0}\right)^{2}\right\} \\
& \times \exp \left\{j \gamma\left(\left(\frac{-N_{x}}{2}+h_{x}-1\right) \Delta x_{0},\left(\frac{-N_{y}}{2}+h_{y}-1\right) \Delta y_{0}\right)\right\} \exp \left\{j \gamma\left(\left(\frac{N_{x}}{2}-1\right) \Delta x_{0},\left(\frac{N_{y}}{2}-1\right) \Delta y_{0}\right)\right\} \\
& \left.\times \exp \left\{j \gamma\left(\left(\frac{-N_{x}}{2}\right) \Delta x_{0},\left(\frac{-N_{y}}{2}\right) \Delta y_{0}\right)\right\} \exp \left\{j \gamma\left(\left(\frac{N_{x}}{2}-h_{x}\right) \Delta x_{0},\left(\frac{N_{y}}{2}-h_{y}\right) \Delta y_{0}\right)\right\}\right] .
\end{aligned}
$$


This can only be 0 if

$$
\begin{aligned}
& -\mathrm{j} \pi \cot \left(\cot \left(\frac{p_{x} \pi}{2}\right)-\cot \left(\frac{q_{x} \pi}{2}\right)\right)\left(-2 h_{x}+2\right)\left(N_{x}-h_{x}\right)\left(\Delta x_{0}\right)^{2}-\mathrm{j} \pi \cot \left(\cot \left(\frac{p_{y} \pi}{2}\right)\right. \\
& \left.-\cot \left(\frac{q_{y} \pi}{2}\right)\right)\left(-2 h_{y}+2\right)\left(N_{y}-h_{y}\right)\left(\Delta y_{0}\right)^{2}-j \gamma\left(\left(\frac{-N_{x}}{2}-1\right) \Delta x_{0},\left(\frac{-N_{y}}{2}-1\right) \Delta y_{0}\right) \\
& \quad+j \gamma\left(\left(\frac{N_{x}}{2}-h_{x}\right) \Delta x_{0},\left(\frac{N_{y}}{2}-h_{y}\right) \Delta y_{0}\right)+j \gamma\left(\left(\frac{-N_{x}}{2}+h_{x}-1\right) \Delta x_{0},\left(\frac{-N_{y}}{2}+h_{y}-1\right) \Delta y_{0}\right) \\
& =\text { Integer } 2 \pi .
\end{aligned}
$$

Since it is extremely unlikely (and can be easily avoided) that all the random values, in the range [0,2 $\pi$, will add in the above equation to produce a multiple of $2 \pi$ we can assume that $D\left(h_{x}, h_{y}\right)$ will never have a zero value and the recursive algorithm can proceed without error. We must remove the random function values and the second dimension to reach the equivalent condition for the phase retrieval method given in [22]

$$
-\mathrm{j} \pi \cot \left(\cot \left(\frac{p_{x} \pi}{2}\right)-\cot \left(\frac{q_{x} \pi}{2}\right)\right)\left(-2 h_{x}+2\right)\left(N_{x}-h_{x}\right)\left(\Delta x_{0}\right)^{2}=\text { multiple } 2 \pi .
$$

This failure condition is guaranteed never to happen [22] if

$$
\frac{\Delta x_{0}^{2} \sin \left(\frac{\left(q_{x}-p_{x}\right) \pi}{2}\right)}{\sin \left(\frac{p_{x} \pi}{2}\right) \sin \left(\frac{q_{x} \pi}{2}\right)}=\frac{1}{2 N} \text {. }
$$

When we extend the algorithm in [22] to a second dimension, the condition for $D\left(h_{x}, h_{y}\right)$ to have a zero value is

$$
\begin{aligned}
& -\mathrm{j} \pi \cot \left(\cot \left(\frac{p_{x} \pi}{2}\right)-\cot \left(\frac{q_{x} \pi}{2}\right)\right)\left(-2 h_{x}+2\right)\left(N_{x}-h_{x}\right)\left(\Delta x_{0}\right)^{2} \\
& \quad-\mathrm{j} \pi \cot \left(\cot \left(\frac{p_{y} \pi}{2}\right)-\cot \left(\frac{q_{y} \pi}{2}\right)\right)\left(-2 h_{y}+2\right)\left(N_{y}-h_{y}\right)\left(\Delta y_{0}\right)^{2}=\text { Integer } 2 \pi
\end{aligned}
$$

which can happen even if the above the orders are set such that Eq. (B.5) (2 above) is held. With the addition of the random phase keys we need not worry about $D\left(h_{x}, h_{y}\right)$ and it is not necessary to impose any relationship between $p_{x}$ and $q_{x}$. To support this claim we note that in over 10000 applications of our software the null case has never occurred.

\section{References}

[1] V. Namias, J. Inst. Maths Appl. 25 (1980) 241.

[2] D. Mendolovic, H.M. Ozaktas, J. Opt. Soc. Am. A 10 (1993) 1875.

[3] H.M. Ozaktas, D. Mendolovic, J. Opt. Soc. Am. A 10 (1993) 2522.

[4] A.W. Lohmann, J. Opt. Soc. Am. A 10 (1993) 2181.

[5] S. Abe, J.T. Sheridan, in: P.C. Cheng, P.P. Hwang, J.L. Wu, G. Wang, H. Kim (Eds.), Focus on Multidimensional Microscopy, vol. 1, World Scientific, Singapore, 1999, p. 1881.

[6] S. Abe, J.T. Sheridan, J. Mod. Opt. 42 (12) (1995) 2373.

[7] L.Z. Cai, Y.Q. Wang, Opt. Laser Tech. 34 (3) (2002) 249.

[8] H.M. Ozaktas, O. Arikan, M.A. Kutay, G. Bozdagi, IEEE Trans. Sig. Proc. 44 (1996) 2141.

[9] C. Candan, M.A. Kutay, H.M. Ozaktas, IEEE Trans. Sig. Proc. 48 (5) (2000) 1329.

[10] P. Refregier, B. Javidi, Opt. Lett. 20 (7) (1995) 767.

[11] B. Hennelly, J.T. Sheridan, Optik 114 (5) (2003) 251.

[12] B. Hennelly, J.T. Sheridan, Opt. Lett. 28 (4) (2003) 269.

[13] G. Unnikrishnan, K. Singh, Opt. Eng. 39 (11) (2000) 2853. 
[14] G. Unnikrishnan, J. Joseph, K. Singh, Opt. Lett. 25 (12) (2000) 887.

[15] G. Unnikrishnan, K. Singh, Opt. Commun. 193 (2001) 51.

[16] S. Liu, L. Yu, B. Zhu, Opt. Commun. 187 (1-3) (2001) 57.

[17] Y. Zhang, C.H. Zheng, N. Tanno, Opt. Commun. 202 (2002) 277.

[18] B. Zhu, S. Liu, Opt. Commun. 195 (5-6) (2001) 371.

[19] N.K. Nishchal, J. Joseph, K. Singh, Opt. Eng. 42 (6) (2003) 1583.

[20] N.K. Nishchal, J. Joseph, K. Singh, Opt. Mem. Neural Net. 12 (2003) 396.

[21] N.K. Nishchal, J. Joseph, K. Singh, Opt. Commun. 217 (1-6) (2003) 117.

[22] W.-X. Cong, N.-X. Chen, B.-Y. Gu, Appl. Opt. 37 (29) (1998) 6906.

[23] X.-G. Xia, IEEE Sig. Proc. Lett. 3 (1996) 72.

[24] O. Matoba, B. Javidi, Opt. Lett. 11 (1999) 762. 